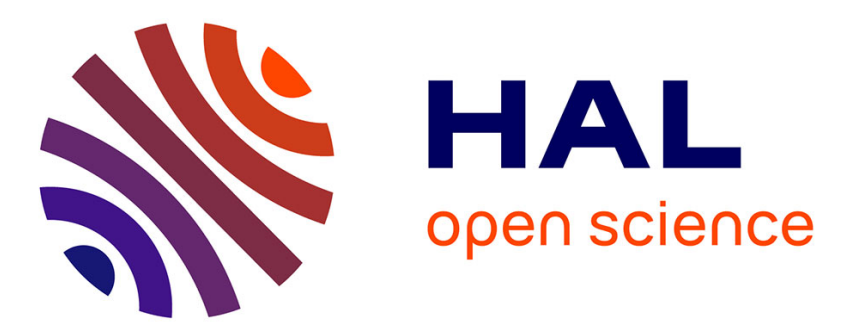

\title{
Travelling and Standing Waves in a Spatially Forced 2D Convection Experiment
}

\author{
François Daviaud, A Burnol, O Ronsin
}

\section{To cite this version:}

François Daviaud, A Burnol, O Ronsin. Travelling and Standing Waves in a Spatially Forced 2D

Convection Experiment . Europhysics Letterss, 1991, 16 (7), pp.667-672. cea-01374013

\section{HAL Id: cea-01374013 https://hal-cea.archives-ouvertes.fr/cea-01374013}

Submitted on 29 Sep 2016

HAL is a multi-disciplinary open access archive for the deposit and dissemination of scientific research documents, whether they are published or not. The documents may come from teaching and research institutions in France or abroad, or from public or private research centers.
L'archive ouverte pluridisciplinaire HAL, est destinée au dépôt et à la diffusion de documents scientifiques de niveau recherche, publiés ou non, émanant des établissements d'enseignement et de recherche français ou étrangers, des laboratoires publics ou privés. 


\title{
HAL \\ archives-ouvertes. ff
}

\section{Travelling and Standing Waves in a Spatially Forced 2D Convection Experiment}

\author{
F Daviaud, A Burnol, O Ronsin
}

\section{To cite this version:}

F Daviaud, A Burnol, O Ronsin. Travelling and Standing Waves in a Spatially Forced 2D

Convection Experiment . Europhysics Letterss, 1991. <cea-01374013>

\section{HAL Id: cea-01374013 \\ https://hal-cea.archives-ouvertes.fr/cea-01374013}

Submitted on 29 Sep 2016

HAL is a multi-disciplinary open access archive for the deposit and dissemination of scientific research documents, whether they are published or not. The documents may come from teaching and research institutions in France or abroad, or from public or private research centers.
L'archive ouverte pluridisciplinaire HAL, est destinée au dépôt et à la diffusion de documents scientifiques de niveau recherche, publiés ou non, émanant des établissements d'enseignement et de recherche français ou étrangers, des laboratoires publics ou privés. 
Europhys. Lett., 16 (7), pp. 667-672 (1991)

\title{
Travelling and Standing Waves in a Spatially Forced 2D Convection Experiment.
}

\author{
F. Daviaud, A. Burnol and O. Ronsin \\ Service de Physique du Solide et de Résonance Magnétique \\ Centre d'Etudes de Saclay - F-91191 Gif-sur-Yvette Cedex, France
}

(received 29 May 1991; accepted in final form 2 September 1991)

PACS. 47.20 - Hydrodynamic stability and instability.

PACS. 47.25 - Turbulent flows, convection, and heat transfer.

\begin{abstract}
Rayleigh-Bénard convection is studied in a rectangular geometry with a spatial forcing induced in one direction by electric wires. When using fluids of relatively large Prandtl numbers, this forcing allows the existence of a perfect one-dimensional pattern until the onset of bimodal convection. The transition to bidimensional convection is studied for increasing Rayleigh number and reveals the existence of different spatio-temporal regimes depending on the value of the forcing. At the onset of the transition, a stationary pattern is observed for weak forcing, while travelling waves are evidenced for strong forcing. Both behaviours give place to collective oscillations at higher Rayleigh number.
\end{abstract}

In the past few years, many studies both experimental and theoretical have been devoted to the problem of the evolution toward turbulent states of convective systems with large aspect ratios [1-4]. However, these systems exhibit defects and display imperfect patterns when the Rayleigh number is increased above the onset of convection. The resulting spatiotemporal regimes are generally very complex and difficult to analyse, except in some particular cases (see, e.g., $[5,6]$ ). On the other hand, the study of one-dimensional periodic systems has recently provided a new approach to the problem of transition to turbulence in weakly confined systems. In these systems, one dimension is larger than the others so that the physical quantities only depend on one space parameter plus time. Related experiments involving Rayleigh-Benard slot convection [7] and theoretical studies of partial differential equations [8] and coupled map chains $[9,10]$ have revealed a transition to spatio-temporal chaos through spatio-temporal intermittency. In order to increase the number of space parameters while keeping a simple spatial structure, interest has also concentrated on the dynamical regimes of two-dimensional (2D) periodic patterns which destabilize when a control parameter is varied. Experiments involving parametrically forced surface waves [11], convection in binary mixtures [12] and in nematic liquid crystals [13] have revealed a richness of pattern formation and dynamical behaviours.

We present here an experimental study of Rayleigh-Bénard convection in a rectangular geometry with a spatial forcing which allows the existence of a perfect $2 \mathrm{D}$ cellular pattern. 
We first describe the experimental set-up and the data acquisition techniques. The different spatio-temporal regimes which are observed when the Rayleigh number $R a$ is increased above the threshold of bimodal convection are then described. We finally analyse and discuss these results.

The experiment was performed in a rectangular cell filled with silicon oil of Prandtl number 30 at $293 \mathrm{~K}$. The vertical side walls were made of plexiglass $(d=5.00 \mathrm{~mm}$ high), while the horizontal plates were made of glass to allow visualization of the convective structures. The rectangular cell had a longitudinal aspect ratio $\Gamma_{x}=L_{x} / d=28$ and a transverse aspect ratio $\Gamma_{y}=L_{y} / d=14$. The temperature difference across the fluid layer was kept constant to within $0.01 \mathrm{~K}$ by means of circulating thermal regulated water. After each temperature increase, dynamical equilibrium was awaited for as long as 24 hours up to 48 hours before each new set of measurement. This time is to be compared with the horizontal diffusion time $\left(\tau_{\mathrm{H}}=L_{x}^{2} / K \approx 20 \mathrm{~h}\right.$, with $\kappa$ the thermal diffusivity).

The spatial forcing was achieved by electric wires regularly disposed parallel to the small side of the cell ( $y$-axis) and near the horizontal hot plate (cf. fig. 1). The distance between them was fixed to $e=2 \mathrm{~cm}=2 \lambda_{c}$, with $\lambda_{c}$ the critical wavelength, at the onset of convection $\left(R a=R a_{\mathrm{c}}\right)$. This gap was chosen because it corresponds to the natural wavelength of the system at the threshold of bimodal convection $\left(R a=R a_{\mathrm{II}}\right)$, when locally measured in the absence of forcing. Experiments realized with a gap $e=\lambda_{c}$ have indeed revealed a mismatch between the forcing and the preferred pattern leading to spatial defects [14]. The tension $U_{0}$ applied to the wires, and thus the forcing, could be varied.

The convective structures were visualized by shadowgraphic imaging. A parallel light beam crossed the cell vertically through the horizontal glass plates. The beam deflection was thus integrated along the cell depth and a top view of the convective pattern was obtained (cf. fig. 3). The spatio-temporal evolution of the convective pattern was recorded using a video camera. The image was digitized in a matrix of $512 \times 512$ pixels with a grey scale of 256 levels (see ref. [7] for details). The data acquisition was generally performed along a line of 512 pixels parallel to the $y$-axis and the acquisition frequency was varied according to the natural frequency of the system.

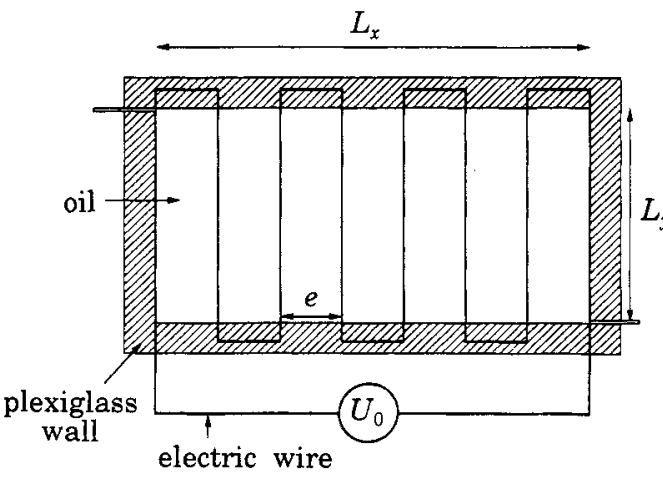

Fig. 1.

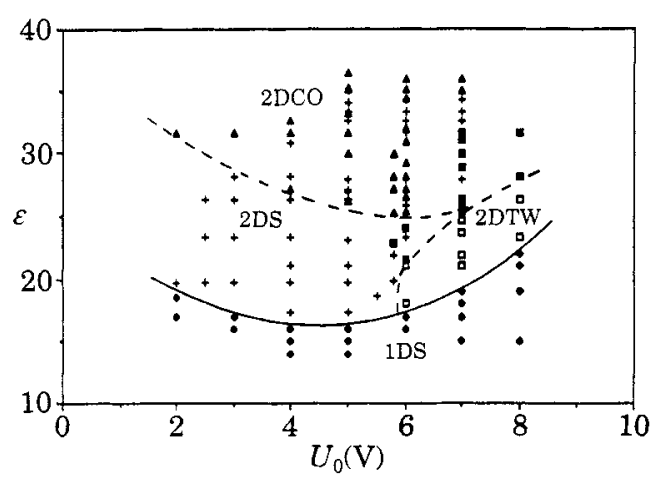

Fig. 2.

Fig. 1. - Qualitative sketch of the experimental apparatus; the fluid is inserted between plexiglass walls and the forcing is achieved by the electric wires, parallel to the small side of the container.

Fig. 2. - Stability diagram of the convective state as a function of $\varepsilon$ and $U_{0} ; 1 D S$ stands for a onedimensional stationary pattern (diamonds), 2DS for a two-dimensional stationary pattern (crosses), 2DTW for travelling waves (open squares) and 2DCO for collective oscillations (triangles); mixed states are represented by dark squares; full lines and dashed lines correspond, respectively, to perfect or imperfect transitions. 
The transition from one-dimensional (1D) convection above $R a_{\mathrm{c}}$ to two-dimensional (2D) convection above $R a_{\mathrm{II}}$ is studied as a function of the two control parameters $U_{0}$ and $\varepsilon=\left(R a-R a_{c}\right) / R a_{c}$. The results are reported on the stability diagram of fig. 2. Bimodal convection appears under the form of second rolls, which start to grow at $R a=R a_{\text {II }}$ and are oriented at right angles with the basic roll pattern. This phenomenon is caused by the instability of the thermal boundary layers and can only be observed in fluids with a relatively large Prandtl number (see ref. [1] for details). First of all, in the absence of spatial forcing $\left(U_{0}=0\right)$, the $1 \mathrm{D}$ convective structure displays defects and instabilities such as the cross-roll and the skewed-varicose instability before the threshold of $2 \mathrm{D}$ convection. The transition to bimodal convection occurs for $\varepsilon \simeq 14$ (the exact value depends on the local wavelength) but the pattern is inhomogeneous and unstable. These observations are in agreement with the results of Busse [1] and Krishnamurti [2] for the corresponding Prandtl number. As $\varepsilon$ is increased further, the two-dimensional pattern destabilizes and spokes are observed (cf. ref. [1]). In fact, even when $U_{0}=0$, the electric wires slightly force the pattern along the $x$-axis and the destabilization first occurs along the $y$-axis (cf. fig. $3 a$ )).

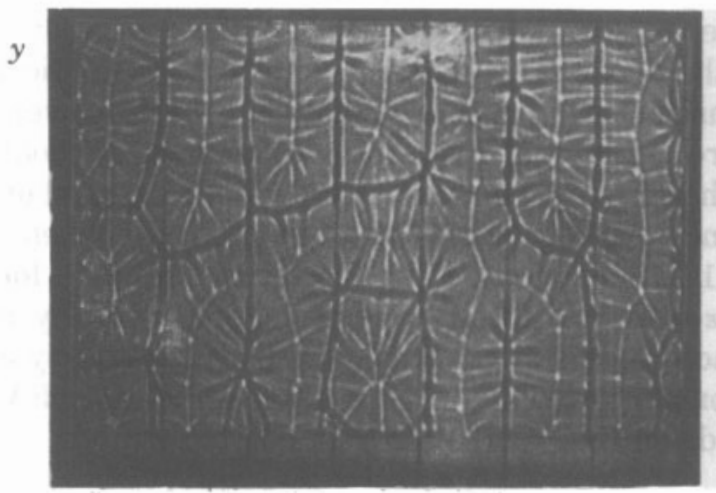

a)

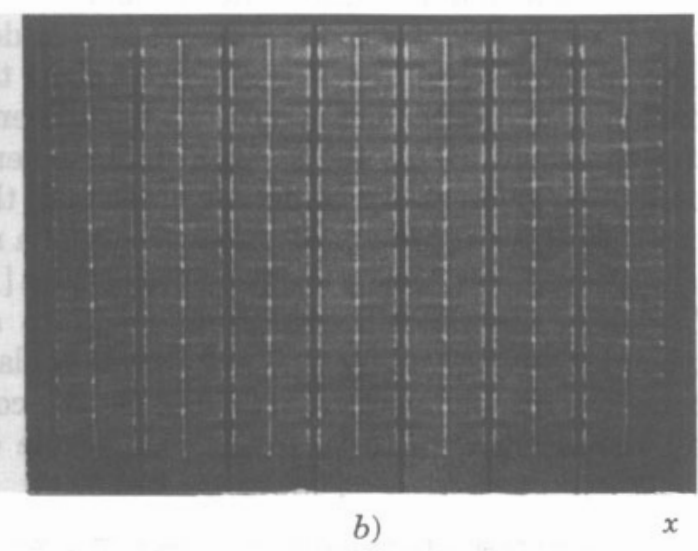

Fig. 3. - Shadowgraphic pictures of the convective structure; a) $U_{0}=0 \mathrm{~V}$ and $\left.\varepsilon=25, b\right) U_{0}=5 \mathrm{~V}$ and $\varepsilon=25$.

On the contrary, the presence of forcing $\left(U_{0} \neq 0 \mathrm{~V}\right)$ allows the existence of a stable and stationary one-dimensional pattern (domain labelled by 1DS in fig. 2) until the onset of bimodal convection $\varepsilon=\varepsilon_{\mathrm{II}}$. The wavelength $\lambda_{x}$ of this first set of rolls is fixed by the electric wires and does not change with $\varepsilon$, at least until $\varepsilon=35$ for $U_{0}=5 \mathrm{~V}\left(\lambda_{x}=2 \lambda_{\text {c }}\right)$. A similar result can be obtained, but only near the onset of convection, by using electric wires at the lateral boundaries [15]. For $\varepsilon>\varepsilon_{\mathrm{II}}$, a second set of rolls appears and a quasi-perfect 2D pattern is observed (cf. fig. $3 b$ )) but its temporal evolution strongly depends on the value of the forcing. As is shown on the stability diagram of fig. 2, a stationary pattern is observed for a weak forcing ( $U_{0}<6 \mathrm{~V}$, domain labelled by $2 \mathrm{DS}$ ), while travelling waves are evidenced for a strong forcing ( $U_{0}>6 \mathrm{~V}$, domain labelled by $\left.2 \mathrm{DTW}\right)$. However, in both cases, the pattern is stable over periods of several horizontal diffusion times $\tau_{\mathrm{H}}$ and no defects are observed. The threshold of bimodal convection $\varepsilon_{\text {II }}$ slightly increases with the forcing $U_{0}$, whereas the wavelength $\lambda_{y}$ of the second set of rolls, which are parallel to the large side of the cell $(x$ axis), decreases when $U_{0}$ or $\varepsilon$ increases $\left(\lambda_{y}=0.78 \lambda_{\mathrm{c}}\right.$ for $U_{0}=4 \mathrm{~V}$ and $\left.\varepsilon=20\right)$. The transition from 1DS to 2DS or 2DTW is well defined and no hysteresis has been observed. When $\varepsilon$ is increased further, both behaviours give place to collective oscillations of the second set of rolls (domain labelled by $2 \mathrm{DCO}$ ). However, the transition from $2 \mathrm{DS}$ or $2 \mathrm{DTW}$ to $2 \mathrm{DCO}$ is imperfect and hysteretic, and mixed states of two different dynamical regimes can be 
observed. In the following, we describe the different spatio-temporal regimes that are observed, depending on the forcing.

In the case of a weak forcing and above $\varepsilon_{\mathrm{II}}$, the $2 \mathrm{D}$ pattern is first stationary for $\varepsilon_{\mathrm{II}}<\varepsilon<\varepsilon_{\mathrm{III}}$ and then displays a collective oscillating mode for $\varepsilon>\varepsilon_{\mathrm{III}}$. The phase of the rolls of the second set oscillates along the direction of the first set of rolls ( $y$-axis) around a mean position with a maximum amplitude $\lambda_{y} / 3$. Hot streams and cold streams oscillate in phase opposition and the frequency $f$ is the same in all the container. This frequency slightly increases with $\varepsilon$ as $\varepsilon^{0.4}\left(f \simeq 0.03 \mathrm{~Hz}\right.$, i.e. $T=1 / f \simeq 0.08 d^{2} / \kappa$ for $\varepsilon=26$ and $\left.U_{0}=5 \mathrm{~V}\right)$. The values of the oscillation frequencies $f$ are in agreement with the results obtained by Busse and Clever [1] and Krishnamurti [2], who have also observed an increase of $f$ with $\varepsilon$, however with a different exponent $\left(f \sim \varepsilon^{23}\right)$. This regime is represented on the spatio-temporal diagram of fig. $4 a$ ). The data acquisition has been performed over 512 pixels along a vertical line parallel to the $y$-axis, between two electric wires and near a cold stream of the first set of rolls. Thus, only the cold streams (bright lines) of the second set of oscillating rolls can be seen in fig. $4 a$ ), but the behaviour of the hot streams is similar. A phase delay can be noticed between the middle of the container and the edges. The behaviour is the same between each couple of electric wires and so there is no $x$-dependence in the amplitude of the oscillations. Only a phase delay has been observed along the $x$-axis. This regime is similar to the optical mode which had been observed in a one-dimensional chain of convective rolls [16]. However, the threshold $\varepsilon_{\text {III }}$ is not so well defined here because of hysteresis and inhomogeneous oscillating regimes can be observed. In fact, the amplitude of oscillation depends on $\varepsilon$ and on the local wavelength $\lambda_{y}$. The appearance of a new pair of rolls in the $y$-direction, e.g. when $\varepsilon$ is increased, can locally stop the oscillations [17]. Such a behaviour can be seen in fig. 2 for $U_{0}=4 \mathrm{~V}$ : when $\varepsilon$ is varied from $\varepsilon=27$ to $\varepsilon=28$, the 2DCO regime (represented by a triangle) corresponding to $\lambda_{y}=0.78 \lambda_{\mathrm{c}}$ is replaced by a stationary regime (represented by a cross) corresponding to $\lambda_{y}=0.70 \lambda_{c}$. On the contrary, a 2DCO regime obtained for $U_{0}=5 \mathrm{~V}$ by increasing $\varepsilon$ up to 30 can remain stable down to $\varepsilon=27$ when $\varepsilon$ is decreased.

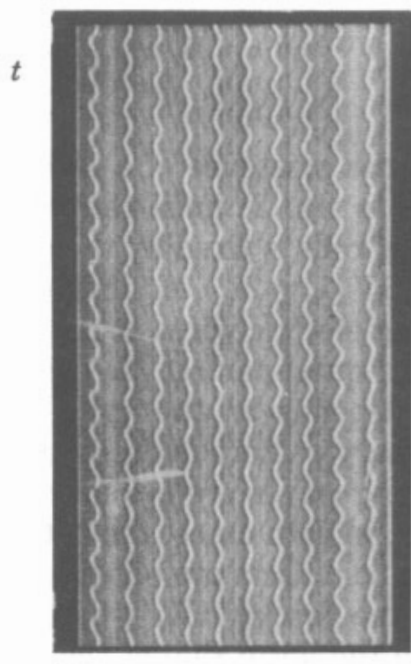

a)

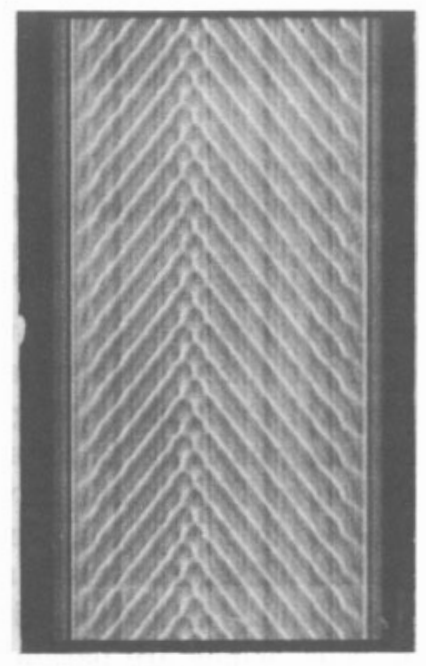

b)

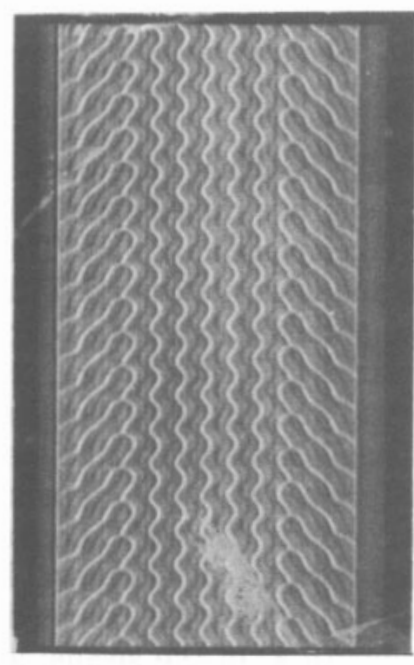

c)

Fig. 4. - Space-times evolution of the convective pattern; spatial digitization is made over 512 pixels along a vertical line parallel to an electric wire and the light intensity is plotted using a grey scale with 256 levels. The total observation time is 512 seconds; a) $2 \mathrm{DCO}$ regime, b) $2 \mathrm{DTW}$ regime, c) mixed state. 
In the case of a strong forcing and for $\varepsilon>\varepsilon_{I I}$, bimodal convection appears as a second set of rolls which propagate along the first set, parallel to the electric wires. More precisely, this regime consists of two waves coming from the large sides of the container (sources) and going to the centre (sink) as is shown in fig. $4 b$ ). The sink is situated near the centre when the two waves the same amplitude but can move near one edge (e.g., the right edge) when the amplitude of one wave (the left wave) is greater than the other. This regime is homogeneous in all the container and the phase velocity $v$ of these waves slightly increases with $\varepsilon$ but does not decrease to zero near the threshold. The corresponding frequency is similar to that observed for the oscillating regime at the same $\varepsilon$ value, except for a small increase due to $U_{0}$. The threshold $\varepsilon_{\text {II }}$ is well-defined and no hysteretic phenomenon has been observed. On the contrary, when $\varepsilon$ is increasing, the transition between 2DTW and 2DCO is complex and mixed states showing simultaneously both propagation and oscillation can be present (cf. fig. $4 c$ )). The same phenomenon occurs when $U_{0}$ is varied and $\varepsilon$ kept constant along this transition line.

In both cases, only collective oscillations were observed above $\varepsilon=35$. The dynamical behaviour then becomes more complex for $\varepsilon>40$. The convective structure displays intermittent oscillations, whose amplitude is a function of space and time, before showing spokes structures and finally entering spatio-temporal chaos.

This study shows that Rayleigh-Bénard convection in a rectangular geometry with a spatial forcing allows the existence of perfect and stable $1 \mathrm{D}$ and $2 \mathrm{D}$ periodic patterns. The similarity between the structure of bimodal convection and a $2 \mathrm{D}$ crystal lattice is striking and leads to study its stability $v s$. the two control parameters. New spatio-temporal regimes have been evidenced. The oscillating mode, which corresponds to a phase oscillation, can be compared with the regime observed by Busse and Whitehead [1], though their experimental procedure is different and only allows the existence of transients. In fact, the patterns studied in ref. [1] are created by controlled initial conditions (illumination of a grid over in an otherwise normal convection container) and are not stable over long time periods. The oscillating bimodal cells thus cannot be generated in a strictly homogeneous pattern. Busse and Clever have performed a stability analysis of the fully $3 \mathrm{D}$ nonlinear problem of bimodal convection [1]. Their numerical results explain the collective oscillation of the bimodal cells in terms of an oscillatory instability and the destabilization of the bimodal pattern to spokes in terms of a knot instability. In the case of a weak forcing, our experimental results seem to agree, at least qualitatively, with this approach. However, the frequencies obtained in their calculations (for a Prandtl number 7) are inferior to our experimental values.

The results concerning the propagation of convective rolls are to our knowledge unknown in Rayleigh-Bénard convection with pure fluids at high Prandtl numbers. They remind us of the results obtained in the study of binary fluid mixtures [18] and oscillatory convection in

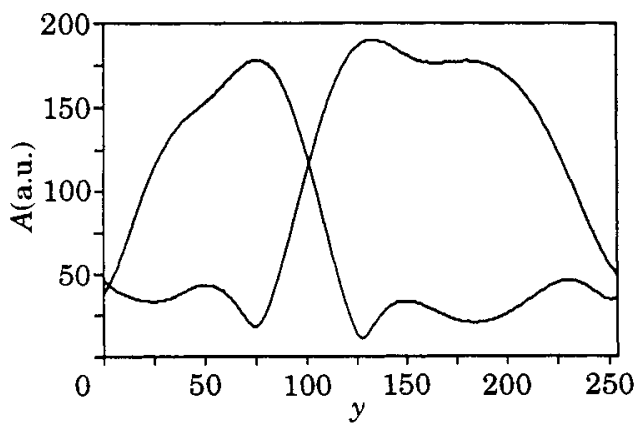

Fig. 5. - Wave amplitude profiles $A$ corresponding to the travelling-wave state of fig. $4 b$ ). 
low Prandtl number fluids [19], though the physical mechanism is different. In fact, this regime can be interpreted within the frame of amplitude equations and work is currently under progress in this direction [17]. By means of complex demodulation techniques (cf. ref. [19]), we have measured the amplitude profiles of the waves observed in the different spatio-temporal regimes. Figure 5 displays the profiles of the left-going and right-going waves corresponding to the travelling waves shown in fig. $4 b$ ). The amplitude of the leftgoing wave is large in the left part of the $y$-axis (source on the left) and decreases down to the sink after which it is dominated by the right-going wave. A phenomenological model based on two coupled Swift-Hohenberg equations is also being developed and the first results look promising [20]. However, the exact role of a local forcing on convection is still unknown and more theoretical work is necessary to explain, for instance, the propagations of rolls.

In conclusion, 2D forced convection exhibits a rich variety of spatial and dynamical behaviours. It shows both stationary and propagative phenomena at the onset of bimodal convection, depending on the values of the control parameters. The possibility to have a perfect $2 \mathrm{D}$ periodic oscillating pattern seems also very interesting to study the transition to spatio-temporal chaos.

We wish to acknowledge G. Balzer, P. Bergé, V. CroquétTe, M. Dubois, and C. Perez-Garcia for fruitful discussions, and P. HEDE and B. Ozenda for their technical assistance.

\section{REFERENCES}

[1] Busse F. H. and Whitehead J. A., J. Fluid Mech., 66 (1974) 67; Busse F. H. and Clever R. M., J. Fluid Mech., 91 (1979) 319; Clever R. M. and Busse F. H., J. Fluid Mech., 198 (1989) 345.

[2] Krishnamurti R., J. Fluid Mech., 42 (1970) 295.

[3] Alhers G. and Behringer R. P., Phys. Rev. Lett., 40 (1978) 712.

[4] Pocheau A., Croquette V. and Legal P., Phys. Rev. Lett., 55 (1985) 1094.

[5] Cross M. C. and Newell A. C., Physica D, 10 (1984) 299.

[6] Pocheau A., J. Phys. (Paris), 49 (1988) 1127.

[7] Daviaud F., Dubois M. and Bergé P., Europhys. Lett., 9 (1989) 441; Daviaud F., BonetTi M. and Dubois M., Phys. Rev. A, 42 (1990) 3388.

[8] Chaté H. and Manneville P., Phys. Rev. Lett., 58 (1988) 112.

[9] Kaneko K., Physica D, 34 (1989) 1.

[10] Chaté H. and Manneville P., Physica D, 32 (1988) 409.

[11] Truffiaro N., Ramshankar R. and Gollub J. P., Phys. Rev. Lett., 62 (1989) 422; Ciliberto S., Douady S. and Fauve S., Europhys. Lett., 15 (1991) 23.

[12] Steinberg V., Moses E. and Fineberg J., Nucl. Phys. B, 2 (1987) 109.

[13] Rehberg I., Rasenat S., Fineberg J., de la Torre Juarez M. and Steinberg V., Phys. Rev. Lett., 61 (1988) 2449.

[14] BALZER G., private communication; for an analysis of spatial resonant forcing in convection, see, e.g., Kelly R. E. and PAL D., J. Fluid Mech., 86 (1978) 433.

[15] Croquette V., Contemp. Phys., 30 (1989) 113.

[16] Dubois M., DaSilva R., Daviaud F., Bergé P. and Petrov A., Europhys. Lett., 8 (1989) 135.

[17] Daviaud F., to be published.

[18] Kolodner P. and Surko C. M., Phys. Rev. Lett., 61 (1988) 842.

[19] Croquette V. and Williams H. L., Physica D, 37 (1989) 300.

[20] Bestehorn M. and Perez-Garcia C., Europhys. Lett., 16 (1991) 225. 